\title{
La concepción heideggeriana del logos de Heráclito
}

\section{Heidegger's conception of heraclitean logos}

\author{
CRISTÓBAL HOLZAPFEL \\ Universidad de Santiago
}

Recibido: 16/06/2017 Aceptado: 23/09/2017

\section{RESUMEN}

Sin duda, la mayor transformación que ha experimentado la humanidad hasta ahora es la del tránsito del mito al lógos. Heráclito descuella como pensador del lógos. La intuición radical que él tiene es que el cosmos es íntegramente lógos, lo que, en cierto sentido, vale como anticipo de la formulación del principio de razón suficiente de Leibniz. Nos atenemos al modo como Heidegger entiende los alcances del lógos, tomando, al mismo tiempo, cierta distancia, sobre todo respecto de Leibniz, ya que, si bien el ser puede ser concebido como lógos, ratio, razón o fundamento, él mismo carece de fundamento, es abismo.

PALABRAS CLAVE:

$$
\text { LÓGOS - PRINCIPIO DE RAZÓN SUFICIENTE - SER - ABISMO }
$$

\begin{abstract}
Doubtless the major transformation that humanity has experienced until now, is the transit from myth to logos. Heraclit stands out as the thinker of logos. The radical intuition that he has is that cosmos is entirely logos, what, we may say, anticipates the principle of sufficient reason of Leibniz. We follow here the way how Heidegger understands logos, taking distance, at the same time, especially from Leibniz, in so far being can be conceived as logos, ratio, reason or foundament, while he himself lacks of foundament, and therefore is abyss.
\end{abstract}

KEY WORDS

LOGOS - PRINCIPLE OF SUFFICIENTE REASON - BEING - ABYSS

(C) Contrastes. Revista Internacional de Filosofia, vol. XXII-N² (2017), pp. 193-201. ISSN: 1136-4076

Departamento de Filosofía, Universidad de Málaga, Facultad de Filosofía y Letras Campus de Teatinos, E-29071 Málaga (España) 
Si POR LO PRONTO dimensionamos el alcance del lógos en la filosofía griega, cómo no, hay que arrancar de recordar que la filosofía comienza con un tránsito del mito al lógos suscitado por ella misma en el proceso de su estado naciente. A su vez, podríamos agregar aquí, con ello nace también un nuevo tipo humano, a saber, el animal racional (independientemente de que este nombre provenga un par de siglos más tarde de Aristóteles). De acuerdo con Wilhelm Nestle y su opera magna Vom Mythos zum Logos, Del mito al lógos, es en primer lugar en Jenófanes de Colofón en que ocurre el mencionado tránsito, y más precisamente en lo que podemos describir como «crítica al antropomorfismo teológico». ${ }^{1}$ En anteriores civilizaciones a la helena el fundamento siguió siempre siendo el mito y la religión, y lo que descuella en Grecia es que, en cierto modo, el fundamento del lógos se ha de imponer, amenguando el mito, pero sin dejarlo fuera de competencia.

Patentemente es ante todo Heráclito el primer pensador del lógos en la filosofía occidental, el que pensó el arjé como lógos.

Se reconocerían en su pensamiento tres niveles del lógos:

Primer nivel: hay un cosmos que está regido por el lógos, es decir, por una legalidad lógico-matemática, de la que nada se sustrae. Agreguemos que si hay un aforismo que el Oscuro de Éfeso nunca escribió, pero está a la base de todos sus aforismos, es que el cosmos es íntegramente lógos.

Cabe aducir que este lógos heraclíteo es sui generis, ya que no expresa una armonía puramente formal, sino que es capaz de aunar los contrarios. En él, el día y la noche son uno, lo mismo vida y muerte, como también incluso bien y mal, justicia e injusticia.

Ateniéndonos a la traducción de Conrado Eggers de la Edición Gredos, el aforismo 50 dice:

«Cuando se escucha, no a mí, sino a la Razón, es sabio convenir en que todas las cosas son una» (Lfp, B 50). ${ }^{2}$

Lo que se expresa en este Fragmento vale a la vez como definitorio de la filosofía: ante todo se trata de escuchar al lógos. Así lo entiende también Heidegger, citándolo e interpretándolo tanto en el artículo Logos de Vortrâge und Aufsâtze (Conferencias y artículos) como en ¿Qué significa pensar? (II Parte) que, junto con Der Satz vom Grund (La proposición del fundamento) y

1 Nestle, Wilhelm, Vom Mythos zum Logos (Del mito al lógos), , Stuttgart: Kröner, 1942, «Einleitung» (Introducción»).

2 Los filósofos presocráticos, Introducciones, traducciones y notas de Conrado Eggers Lan y Victoria E. Julia, Madrid: Gredos, 1986, vol. 12. 
su Lección final, la Lección XIII, constituyen los principales referentes de los alcances del logos en el pensamiento heideggeriano.

Esto uno (el lógos) reúne los contrarios:

«Guerra es padre de todos, rey de todos: a unos ha acreditado como dioses, a otros como hombres: a unos ha hecho esclavos, a otros libres» (Lfd, B 53).

«El camino hacia arriba y hacia abajo es uno y el mismo» (Lfd, B 60).

Y así como prematuramente en el inicio mismo de la filosofía occidental se observan rasgos teológico-negativos, a saber, ya en el propio Jenófanes de Colofón, así también en el Fragmento 32 en atención a la justificación de llamar y no llamar a lo Uno (el lógos) con el nombre de Zeus:

«Uno, lo único sabio, quiere y no quiere ser llamado con el nombre de Zeus» (Lfp, B 32).

El segundo nivel corresponde al logos cósmico universal y que equivale a su manifestación física. Esta corresponde al fuego ( $p y r)$ como el que hay en el sol y las estrellas.

«Este mundo, él mismo para todos, ninguno de los dioses ni de los hombres lo ha hecho, sino que existió siempre, existe y existirá en tanto fuego siempre vivo, encendiéndose con medida y con medida apagándose» (Lfp, B 30).

El tercer nivel refleja como ese lógos nos determina a nosotros como seres humanos, ya que justamente lo que nos define es que somos esencialmente racionales.

Como observamos, somos poseedores de lógos en la medida en que estamos determinados por el lógos cósmico universal. En ello se manifiesta el cosmocentrismo que, en general, es característico de la filosofía griega.

Pero ¿somos poseedores de lógos, como reza el zoón lógon éjon aristotélico, o estamos más bien poseídos por el lógos, como sería probablemente en Heráclito?

Con Aristóteles, la vista sería el órgano de los sentidos más abarcante. Consideremos todas las diferencias que hacemos a través de la vista. Teniendo presente la Biología, y más precisamente la Oftalmología, podríamos decir, que en todo momento están activadas en la visión más de mil funciones. La vista, aparte de su función más propia (ver) tiene además relación con el dominio, incluso diríamos un dominio territorial del entorno en que se mueve el animal depredador con la focalización de su mirada, dominio que, a su vez, se expresa en mandatos, en órdenes que suele dar el amo, el señor o la autoridad nada más que con la mirada. El oído, en cambio, está directamente ligado a la capacidad 
de hablar, y por consiguiente al lenguaje, y junto con ello, a la música, y la cultura en su más amplio alcance. ${ }^{3}$

En la mencionada Lección XIII de la obra de Heidegger sobre el principio de razón suficiente, basado en un seminario dictado en la Universidad de Friburgo en 1956, sostiene que el griego lógon didonai dice algo muy distinto de que el latino reddere rationem, traducido usualmente como 'dar cuenta de algo', y que está directamente relacionado con los alcances del principium rationis sufficientis de Leibniz en plena modernidad. El lógon didonai Heidegger lo entiende más bien en el sentido de un traer a la presencia, de un sacar a luz el fundamento, y junto con ello, el ser de algo. Ello nos muestra que desde antiguo ser y fundamento van juntos, están en una identidad inseparable. Dice Heidegger a propósito de esto:

«En tanto cada ente está determinado por el ser, esto es, por el fundamentar, el ser es cada vez algo fundamentante y fundamentado, y esto de acuerdo a los distintos modos, cuya multiplicidad y origen no pueden ser abordados aquíı. ${ }^{4}$

Y agrega más adelante:

«Como lógos es el ser lo primero, desde dónde lo presente se presenta, en griego to próton ozen. «Lo primero desde dónde» es aquello, desde dónde cada cosa, que es, se inicia /anfângt/, y desde dónde permanece como lo inicial dominante; iniciar mienta en griego arjéin. El lógos se despliega así como proton ozen, vale decir, como arjé, dicho de modo latino-romano, como principium».

Tengamos en cuenta que lógos, y en su traducción latina, ratio, admite, entre otros, dos posibilidades de traducción: como razón y como fundamento, diciéndose este último en alemán: Grund, y por eso la expresión del principium rationis sufficientis, en este idioma es: Der Satz vom Grund.

Sin embargo, en la modernidad asistimos a un divorcio entre entre ser y lógos, y más precisamente, entre ser y ratio, entre ser y fundamento (Grund). Desde la perspectiva heideggeriana, en la modernidad se ha acentuado máximamente el olvido del ser, asistimos a una separación del ámbito representacional de lo que debería ser su origen, esto es, el ser.

3 Al respecto, Rivera, Jorge Eduardo, Itinerarium cordis, Santiago: Brickle Ediciones, 2006, IV «La riqueza del sentir».

4 Heidegger, Martin, Der Satz vom Grund, Pfullingen: Neske, 1971. Éste y demás textos citados son todos de la «Lección XIII», y la traducciones son mías. / Ed. cast.: La proposición del fundamento, trad. de Felix Duque y Jorge Pérez de Tudela, Barcelona: Serbal-Guitard, 1991. 
En ello se muestra cierta coincidencia con el pensamiento de Michel Foucault de Las palabras y la cosas, y que concierne a lo que se considera ahí como la segunda episteme en su «arqueología del saber», vale decir, la que tiene que ver con la instauración de un espacio representacional, en el cual se establecen las condiciones para que, en cierto modo, algo tenga «derecho a ser», como espacio-tiempo, aceleración, velocidad, masa, volumen, etc., teniendo en cuenta ciertas variables de la Física moderna. ${ }^{5}$ Recordemos que en la episteme anterior, llamada «prosa del mundo», los signos estaban más bien alojados en las cosas, razón por la cual había que sumergirse en ellas para poder descifrarlas, incluyendo ello también, la posibilidad de desentrañar ciertos mensajes ocultos, como lo hacían en aquella era la mántica, la cábala, la videncia, la adivinanza, el vaticinio, la interpretación de vuelos o trinos de aves, y otros. Expresado esto mismo con Heidegger:

«Cuando modernamente el ser es determinado trascendentalmente como objetualidad /Gegenständigkeit/ y ésta como condición de posibilidad del objeto, entonces desaparece a la par el ser en favor de aquello que se llama condición de posibilidad y que es de la naturaleza del fundamento racional y del fundamentar».

Pues bien, si el lógos heraclíteo, aunque sea siguiendo el derrotero del olvido del ser, va a replantearse en el principio de razón suficiente de Leibniz, en el siglo XVII, partamos ahora por ver los alcances de este principio. En una de sus tantas expresiones en la obra de Leibniz, leemos en la Teodicea, I Parte, parágrafo 44:

«/.../ principio de razón suficiente: que nunca acontece algo sin una causa o siquiera una razón determinada, esto es, sin una cierta razón a priori, por qué existe algo y no más bien no existe y por qué existe más bien de éste que de ningún otro modo. Este importante principio vale para todos los acontecimientos, y no se deja aducir ninguna prueba contraria».6

Distinguimos tres estadios del principio de razón suficiente:

1. Estadio ontológico, de acuerdo al cual todas las cosas, el universo íntegro, se comporta como se comporta de acuerdo a este principio. Supongamos un huracán que se forma, cómo se agranda, su desplazamiento, hay razones

5 Foucault, Las palabras y las cosas, trad. de Cecilia Frost, Madrid: Siglo XXI, 1989, capítulo «Representar».

6 Leibniz, Theodizee, Edit. Insel, Frankfurt am Main, 1986, I Parte, \# 44, trad.m./ Ed. cast.: Teodicea, \# 44, en: Obras, Tomo V, trad. de Patricio Azcárate, Casa Editorial de Medina, s/a. 
para ello, dadas por las presiones, la temperatura, la época del año, la región geográfica, y demás. Y lo mismo todo cuanto sucede.

En este estadio el principio, la ratio, el fundamentum, está en las cosas mismas al modo del lógos o razón que determinan que ellas sean como son.

2.Estadio epistemológico, de acuerdo al cual el principio determina el saber, el conocimiento y la ciencia, en cuanto a un volver a dar la razón suficiente o fundamento a lo que de por sí lo tiene en ello mismo. Si un científico explica que las fases de la luna se deben a su cambio de posición con respecto a la tierra y el sol, le vuelve a dar la razón - al menos suficiente - a ese fenómeno que, de por sí, la tiene de antemano. En otras palabras, no porque el científico de con esa razón suficiente la está creando, sino que la luna está desde tiempos inmemoriales mostrándose en distintas fases.

Si en el estadio ontológico el principio actúa como fundamento que está en las cosas mismas y su entorno (el agua que se desplaza como se desplaza, el sonido que se comporta como se comporta, la luminosidad que es la tiene que haber en cualquier lugar y momento del Planeta o del universo en plenitud), el estadio epistemológico es más bien el de la fundamentación (cómo fundamenta el ser humano que la luminosidad es la que tiene que haber aquí o acullá, etc.).

La mencionada fundamentación, en relación con el estadio ontológico del principio de razón suficiente, es en rigor un volver a dar el fundamento a lo que de por sí lo tiene en ello mismo. Por ejemplo, tales y cuales son las razones de acuerdo a las cuales hay aquí y en este momento la presión que hay, y por supuesto habría que señalar exactamente esas razones: frente frío o caliente en el Océano, vientos que van en tal dirección y de tal velocidad, etc.

3.Estadio existencial. Si decidimos, si hacemos, si evitamos algo, si nos interesa, nos motiva, nos inquieta, nos gusta o nos disgusta algo, en todo ello nos regimos por razones que deberían ser suficientes para ello. Aquí sólo relativamente hay un fundamento previo en las cosas, en cuanto a que, por ejemplo, evaluando cierta situación dada, tomamos una decisión. Éste es el motivo por el cual no hay aquí tampoco un volver a dar el fundamento, sino un simple dar el fundamento. Y agreguemos que sólo podemos dar propiamente el fundamento o razón, sobre la base de la libertad. ¿O no será al revés? Que, justamente somos libres porque podemos dar el fundamento a lo que sea y, digamos además, de modo variado, y hasta por capricho, antojo, porfía. Si fuera así, el estadio existencial constituiría a la vez una definición de la libertad del ser humano de hacer esto o lo otro.

Pero, por decirlo así, y de modo capcioso, así como el color rojo no es rojo, así tampoco la libertad es libre. Al ser ella expresión también del principio de razón suficiente en su estadio existencial, ella requiere precisamente de principios, de razones, de fundamentos para validar lo que decidimos, pensamos e incluso recordamos, imaginamos y sentimos. 
En el pensamiento de Heidegger detectamos al menos dos teorías del poder - que asunto aparte, habría que ver cómo con se concilian y complementan o no, una con otra: la primera la encontramos en Ser y tiempo y tiene el alcance de que el «se», el «man», el «se dice lo que se dice», «se hace lo que se hace», y otros, que rige sobre la cotidianidad. En cierto modo, el poder está aquí despersonalizado, porque es «todos», pero «nadie» a la vez; es simplemente el «se». Éste «prescribe la intepretación próxima del mundo y del ser-en-el mundo». ${ }^{7}$

La segunda teoría remite a La proposición del fundamento, ya que el poder es aquí el del propio principio de razón suficiente que está detrás de toda forma particular de poder. Y este «poder» se explaya más precisamente como zustellen, 'emplazar'. El principio, en cierto modo, «esgrimido» por cada cual, permite demandar de cada ente por qué es como es y se comporta como se comporta.

Pero, agreguemos, habría otra forma, más radical y ontológicamente primaria de poder, y que es la propia del estadio ontológico del principio de razón suficiente, y que, por supuesto, el autor de este principio es la que más subraya, a saber, que el poder es el lógos mismo «en las cosas», como que éstas en todo lo que hacen, cómo cambian, cómo se mueven, se transforman, y otros, todo ello está enteramente regido por el lógos. Advirtamos que esto nos permite advertir el carácter absoluto del lógos. Los entes, las cosas, el mundo, todo lo que acontece, es lo que es, debido a él, siendo cada cosa en cierto modo su resultado, en buenas cuentas, el resultado de su determinación. Si el lógos sería de este modo «omnipotente», en ello radica también la explicación de su divinización, que ya se encontraría en el Evangelio de San Juan, y más tarde en distintos momentos de la filosofía medieval.

Pero, con toda la omnipotencia del ser en tanto fundamento, ratio, lógos, y así se presenta en el pensamiento leibniciano, para Heidegger más bien se trata de que es propio del ser retirarse (sich entziehen). El ser supone no sólo revelación (Offenbarung), sino a la vez retiro (Entzug), y esto atañe por supuesto tanto al ser mismo, al ser de la plenitud, como al ser de cada cosa. Tanto de una persona como de una naranja, podemos decir que a la vez que se nos revelan, se nos ocultan, siendo el ser de ellas pues también retiro, y ello no como algo deliberado (como podría ser en el caso de una persona, porque es tímida, inhibida o introvertida, por ejemplo), sino porque el ser es siempre retiro. Heidegger:

7 Heidegger, Martin, Sein und Zeit, Tübingen: Niemeyer, 1977, parágrafo 27, p. 129. / Ed. cast.: Ser y tiempo, trad. de José Gaos, México: FCE, 1962. Tb.: Ser y tiempo, trad. de Jorge E. Rivera, Santiago: Universitaria, 1997. 
«Ocasionalmente en lo que nos condujo a decir claramente lo que quiere decir la historia del ser como la historia del destino del ser /Seinsgeschick/, se apuntó a que el ser en tanto se nos destina /uns zuschickt/y esplende /lichtet/ al mismo tiempo, se retira. El discurso del retiro permaneció oscuro y sonó para algún oído como una afirmación mística, no apoyada en nada concreto. Ahora podemos escuchar más claramente la palabra del retiro del ser. La palabra dice: el ser se oculta /verbirgt/ como ser, a saber, en su inicial /anfänglich/ copertenencia con el fundamento como lógos».

El aludido retiro del ser como fundamento, podríamos decir que lo desfonda, lo deja sin fundamento. Con Heidegger se trata de que si todo tiene fundamento para ser lo que es y comportarse como se comporta, menos el propio fundamento. El fundamento es sin fundamento, es abismo. Con ello se descubre el abismo del ser /Abgrund des Seins/. Heidegger:

«En tanto el ser se esencia /west/ como fundamento, él mismo carece de fundamento. Y esto, sin embargo, no porque se fundamente a sí mismo, sino porque cada fundamentación, también y precisamente aquella por sí mismo, no se le ajusta al ser como fundamento. Cada fundamentación, y cada apariencia de fundamentabilidad rebajaría al ser a algo ente. El ser queda como ser sin-fundamento lgrund-los/. Del ser queda el fundamento, esto es, como un fundamento recién fundamentante, fuera y aparte /weg und ab/. Ser: el a-bismo /Ab-grund/».

Digamos finalmente que mucho más en concordancia con la concepción del ser humano como «pastor del ser», el que cuida el ser, y que no está más empeñado en el dominio («El hombre no es más el Señor de la Tierra, sino el pastor del ser»), como lo expresa el pensador de la Selva Negra en la Carta sobre el «humanismo», está el alcance del ser como abismo y al mismo tiempo la escucha del lógos del aforismo 50 de Heráclito.

Mas, se trata por supuesto de que estas palabras no han sido oídas, y si hasta cierto punto si fueron oídas en la filosofía, no han irradiado hacia fuera, de tal manera que lo que prima es el empoderamiento impresionante que le brinda al ser humano el lógos derivado en ratio sufficiens y el consiguiente emplazamiento de todo ente.

Cristóbal Holzapfel es profesor titular de la Universidad de Chile.

Líneas de investigación:

Historia de la filosofía contemporánea; filosofía alemana; Antropología filosófica

Últimas publicaciones:

Ser Humano (Cartografía antropológica), Cinta de Moebio Ediciones, Santiago de Chile, 2014. 
Argumentación y proyección de mundo, Editorial Universitaia, 2015.

Email: hcristob@gmail.com 
